\title{
Motivos de uso y no uso de puentes peatonales en la Ciudad de México: la perspectiva de los peatones
}

\author{
Elisa Hidalgo-Solórzano, M en C, (1) Julio Campuzano-Rincón, Dr en C, ${ }^{(2)}$ Jorge M Rodríguez-Hernández, M en C, DSc, ${ }^{(3)}$ \\ Luis Chias-Becerril, PhD, (4) Héctor Reséndiz-López, PhD, (4) Harvey Sánchez-Restrepo, PhD, (4) \\ Bernardo Baranda-Sepúlveda, M en C, ${ }^{(5)}$ Claudia Franco-Arias, Ing Transp, ${ }^{(5)}$ Martha Hijar, Dr en C. ${ }^{(2)}$
}

\author{
Hidalgo-Solórzano E, Campuzano-Rincón J, \\ Rodríguez-Hernández JM, Chias-Becerril L, \\ Reséndiz-López H, Sánchez-Restrepo H, \\ Baranda-Sepúlveda B, Franco-Arias C, Hijar M. \\ Motivos de uso y no uso de puentes peatonales \\ en la Ciudad de México: la perspectiva de los peatones. \\ Salud Publica Mex 2010;52:502-510.
}

\begin{abstract}
Resumen
Objetivo. Analizar los motivos de uso y no uso de puentes peatonales (PP). Material y métodos. Se empleó un diseño transversal, a partir de una muestra de peatones usuarios y no usuarios de PP; se utilizó regresión logística para identificar los factores que influyen en el uso y no uso de PP. Resultados. La prevalencia de no uso fue $50.5 \%$ en 813 peatones entrevistados; la principal razón para usar PP fue "seguridad", del no uso fue "flojera". Hubo diferencias significativas al analizar motivos de no uso de PP en las edades de 19 a 36 años, ajustando por escolaridad y características físicas del PP, con RMa. I.7 (IC95\% I.06-2.86) y RMa. I.9 (IC95\% I.I43.33), respectivamente. Conclusiones. Los resultados de este estudio permiten identificar aspectos importantes a considerar desde la perspectiva de los peatones, antes de construir nuevos PP, así como aquéllos que deben mejorarse para incrementar su uso en zonas de alto riesgo de lesiones por atropellamiento.
\end{abstract}

Palabras clave: prevención de accidentes; accidentes de tránsito; puentes; lesiones; México

\author{
Hidalgo-Solórzano E, Campuzano-Rincón J, \\ Rodríguez-Hernández JM, Chias-Becerril L, \\ Reséndiz-López H, Sánchez-Restrepo H, \\ Baranda-Sepúlveda B, Franco-Arias C, Híjar M. \\ Use and non-use of pedestrian bridges \\ in Mexico City: the pedestrian perspective. \\ Salud Publica Mex 2010;52:502-5I0.
}

\begin{abstract}
Objective. To analyze the motives for using and not using pedestrian bridges (PB). Material and Methods. A crosssectional survey was conducted of a sample of pedestrian users and non-users of PB; a logistic regression model was used to analyze the motives for use and non-use. Results. The prevalence of non-use was $50.5 \%$ of 813 surveyed pedestrians; the principal reason to use a PB was safety, and not to use it was "laziness". There were significant differences when analyzing the reason of non-use in the age groups 19 to 36 years, adjusted for education and physical characteristics of the PB ([aOR=I.7; $95 \% \mathrm{Cl}=1.06-2.86]$ and [ORa.I.9; 95 $\% \mathrm{Cl}=$ I. 14-3.33], respectively). Conclusions. The results of this study allow us to identify important aspects to consider -from the perspective of the pedestrians- when constructing new $P B$ and improving existing $P B$ to increase use in areas with a high risk of pedestrian injuries.
\end{abstract}

Key words: accident prevention; accidents, traffic; bridges; injuries; Mexico

(I) Centro de Investigación en Sistemas de Salud. Instituto Nacional de Salud Pública. Cuernavaca, Morelos, México.

(2) Centro de Investigación en Salud Poblacional. Instituto Nacional de Salud Pública. Cuernavaca, Morelos, México.

(3) Escuela de Salud Pública de México. Cuernavaca, Morelos, México.

(4) Instituto de Geografía, Universidad Nacional Autónoma de México. México DF, México.

(5) Instituto de Transporte y Desarrollo de Políticas. México DF, México.

Fecha de recibido: 15 de febrero de 2010 - Fecha de aceptado: 17 de agosto de 2010 Solicitud de sobretiros: Martha Hijar Medina. Centro de Investigación en Salud Poblacional, Instituto Nacional de Salud Pública. Av. Universidad 655, col. Santa María Ahuacatitlán. 62100 Cuernavaca, Morelos, México. Correo electrónico: mhijar@correo.insp.mx 
$\mathrm{A}_{\mathrm{u}}^{1}$ construir una carretera o una vía rápida en zonas urbanas se dividen espacios para dar prioridad al movimiento de vehículos motorizados, y se fragmentan zonas altamente pobladas, sin considerar que sus habitantes tienen la necesidad de movilizarse de un punto a otro, en algunos casos siempre a pie y cargando mercancías. ${ }^{1}$ La solución a ello ha sido, en países como el nuestro, la colocación de puentes peatonales (PP). Este tipo de medidas han tenido las siguientes repercusiones: i) la mayoría de veces se tiene que caminar hasta donde se encuentran los puentes, ii) se percibe que la mayoría de ellos no están ubicados en los sitios más útiles, iii) tienen un diseño muy poco amable para la mayoría de los usuarios, y iv) su utilización generalmente implica más tiempo, esfuerzo y, en algunos casos, mayor inseguridad. ${ }^{2}$

Esta situación provoca que el peatón termine cruzando las calles en los lugares que considera más convenientes, sin importar el hecho de tener que enfrentarse a situaciones de alto riesgo o de riesgo agregado. Por lo anterior, cuando las modificaciones a la vía pública se realizan sin tomar en cuenta a todos los actores que la transitan, se convierten en el espacio más peligroso para el peatón, uno de los usuarios más numerosos y vulnerables de la vía pública. ${ }^{2}$

En este tema, durante el año 2000 se realizó una investigación en la Ciudad de México que buscaba identificar los determinantes de las muertes por atropellamientos, donde los siguientes fueron algunos de los resultados más importantes: ${ }^{3-5}$

- La mayoría eran hombres en edad productiva, mientras que en el caso de las mujeres, éstas pertenecían al grupo de adultos mayores, con limitaciones visuales y motoras para poder desplazarse con seguridad, en un espacio que tradicionalmente no les ha sido familiar, como la vía pública.

- La mayoría de los atropellamientos ocurren en avenidas grandes o en vías de alta velocidad, con gran circulación de vehículos, sin semáforos y con puentes peatonales. En la mayoría de los casos, se trató de lugares muy cercanos al lugar de residencia de los peatones atropellados.

- La mayor parte de los atropellamientos ocurrieron en fin de semana, durante las horas de la noche y, en el caso de los peatones de sexo masculino, un poco más de la mitad había ingerido bebidas alcohólicas.

- Se trata de población que no sabe manejar un automóvil o que no maneja con regularidad, por lo que les resulta muy difícil poder calcular la velocidad real de un vehículo. El 90\% de los casos reportó no saber conducir, y un porcentaje muy bajo afirmaron conocer bien las señales de vialidad.
- $\quad$ Si bien los puentes peatonales se aceptan como elementos útiles, la mayoría de los peatones atropellados admitió no utilizarlos nunca o hacerlo ocasionalmente.

Al ser los atropellamientos la principal causa de muerte accidental en la Ciudad de México, ${ }^{5}$ resulta paradójico observar que los puentes peatonales han sido intervenciones dirigidas a incrementar el flujo vehicular de las vialidades sobre las que se encuentran, más que constituir una medida para prevenir atropellamientos. ${ }^{2}$ El presente trabajo es parte de un estudio realizado en la Ciudad de México durante 2008, en el que además se realizó una evaluación de la estructura y el estado físico actual de los puentes peatonales, y se georreferenciaron las lesiones registradas por atropellamientos. El objetivo de este artículo fue conocer y analizar los motivos de uso y no uso de los puentes peatonales.

\section{Material y métodos}

Se utilizó un diseño transversal en el que se incluyeron peatones usuarios y no usuarios de puentes durante el periodo comprendido entre el 22 de enero y el 1 de febrero de 2008. Se hizo necesaria la obtención de una muestra representativa de puentes peatonales según tipo y delegación donde se encuentran ubicados. Por lo anterior y ante las limitaciones de información previa se decidió aplicar el siguiente esquema de muestreo:

En una primera etapa se determinó una muestra del total de puentes peatonales $(\mathrm{N}=617)$, teniendo en cuenta tres características principales:

- Que representara a todos los tipos de puente (según la vialidad sobre la que se encuentran ubicados: eje vial, avenida primaria, avenida secundaria, etc.)

- Que incluyera tanto a los que tienen como a los que no tienen paso de peatones a nivel de la calle, y

- Que fueran representativos de la distribución según delegación, y por lo tanto del DF.

El tamaño de la muestra de puentes fue de 102. Esta muestra se seleccionó de manera aleatoria, manteniendo la distribución original (peso porcentual) de las tres características antes explicadas. Teniendo en cuenta que la pregunta que se deseaba responder era: ¿Cuáles son los factores que determinan el uso de los puentes peatonales con que cuenta la Ciudad de México?, se consideró que dicho tamaño permitiría detectar diferencias en las proporciones de uso y no uso de los puentes peatonales, de 0.14 con un poder de $81 \%$ y un nivel de confianza de $95 \%$ (figura 1). 


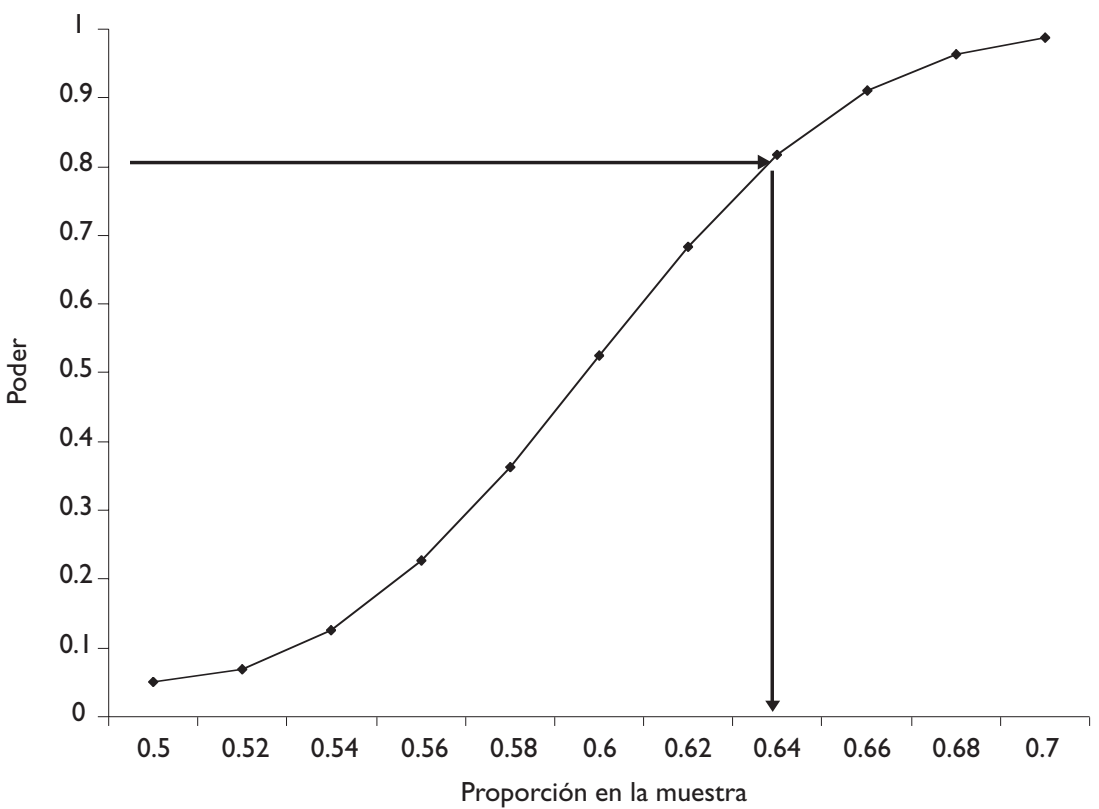

Ho: $\mathrm{p}=0.500$, proporción en la población

Figura I. Curva de poder

En una segunda etapa fue necesario determinar el tamaño de muestra de peatones usuarios y no usuarios de los puentes seleccionados, por lo que se decidió hacerla en campo con el fin de obtener una muestra representativa de los mismos. Para los cálculos de muestra se utilizó la fórmula para proporciones en poblaciones finitas descrita por Dawson y autores afines. ${ }^{6-8}$ Para esto se realizó observación directa a diversas horas del día, empleando las técnicas de conteo y filmación con una duración de 30 minutos por cada puente, contabilizando los usuarios y no usuarios. Debido a la dificultad de conocer la edad exacta se decidió agruparlos en niños, jóvenes, adultos y adultos mayores, todas distribuidas por sexo. Una vez definida la distribución de esta observación, se introdujeron los datos a un programa previamente diseñado en Excel, en el que se consideraron las siguientes características: tipo de puente, con o sin posibilidad de paso a nivel de calle, probabilidad de uso, número de usuarios y no usuarios, sexo y grupo de población. Esta observación permitió además hacer un registro de usuarios y no usuarios, por grupo poblacional y sexo para cada tipo de puente, en el que se observó un volumen de 6436 peatones, de los cuales $79.3 \%$ eran usuarios y $54.1 \%$ fueron hombres.
Con estos datos se obtuvo el tamaño de muestra por tipo de usuario, grupo poblacional y género, para cada puente, con base en la fórmula siguiente:

$$
n=\frac{N z_{\alpha / 2}^{2} P(1-P)}{(N-1) e 2+z_{\alpha / 2}^{2} P(1-P)}
$$

La diferencia del error estimada fue de 0.02 .

El tamaño de muestra estimado fue 2533 peatones, la cual nos permitió obtener un poder de $85 \%$ y un nivel de confianza de $95 \%$. Se permaneció en el lugar durante el tiempo necesario hasta completar la muestra estimada para cada puente.

La encuesta rápida aplicada a peatones, usuarios y no usuarios de los puentes, previo consentimiento informado verbal, contenía las siguientes variables:

- Edad en años cumplidos

- Sexo masculino o femenino

- Escolaridad: último año de estudios

- Ocupación: actividad económica a la que se dedica

- Lugar de nacimiento: municipio donde nació

- Lugar de residencia: nombre del municipio, barrio o colonia donde vive 
- Tiempo de vivir en la Ciudad de México: meses, años

- Forma de transporte más utilizada: auto privado, camión, metro, taxi, a pie

- ¿Generalmente por dónde cruza las calles?: por las esquinas, por el sitio más cerca de donde está, por el puente peatonal (si lo hay), por la cebra o sitio marcado para paso del peatón

- ¿Por qué usa el puente?: pregunta con respuesta abierta, que después se categorizó con base en ésta.

- ¿Cuándo usa el puente peatonal?: pregunta con respuesta abierta, que después se categorizó con base en ésta.

- ¿Por qué no usa el puente?: pregunta con respuesta abierta, que después se categorizó con base en ésta

- ¿Qué propondría para que síse utilizaran los puentes?: pregunta con respuesta abierta, que después se categorizó con base en ésta

La recolección de información se realizó por parte de encuestadores previamente estandarizados, con base en una prueba piloto que también se empleó para probar y validar el instrumento de recolección de información. Por razones de seguridad, la información sólo fue recolectada durante turnos matutino y vespertino.

Se calcularon medidas de tendencia central y de dispersión, para las variables continuas y análisis de varianza para determinar diferencias entre grupos, y frecuencias y porcentajes para las variables categóricas. Se usó la prueba de ji cuadrada para comparar la proporción de peatones que usaron los PP con los que no los usaron, por las diferentes categorías de variables independientes, y así se determinó la existencia de diferencias estadísticamente significativas con un valor $p<0.05$. Se analizaron las razones de uso y no uso de puentes peatonales estratificadas por sexo.

Para analizar los motivos de uso y no uso de puentes peatonales se realizó un análisis de regresión logística, donde la variable dependiente se categorizó como 0 cuando el peatón "usó el puente peatonal" y 1 si el peatón "no usó el puente peatonal". El modelo se definió utilizando el método paso por paso y se ajustó controlando las variables potencialmente confusoras y posibles interacciones. Las variables incluidas en el modelo final fueron edad, categorizada en cuatro grupos (menores de 19 años -percentil 10-, de 19 a 36 -percentil 50-, 37 a 45 -percentil 70- y 46 y más); escolaridad, en cuatro categorías (sin instrucción y primaria, secundaria, bachillerato, licenciatura y posgrado), y característica física de los PP. Esta última variable fue resultado de un aforo realizado a los puentes seleccionados, que incluyó la medición de cada uno de los componentes de la estructura del puente (gálibo o altura, ancho de pasarela, ancho de escalones, huella y contrahuella), evaluación del estado de barandales, malla protectora, iluminación, existencia de basura tanto en la pasarela como en las escaleras, así como existencia de cables de electricidad de alto voltaje. Para obtener la calificación de la característica física del PP, se asignó el valor de " 1 " a cada componente cuando cumplía con los atributos recomendados y "0" (cero) cuando no cumplía. Se hizo una sumatoria de los puntajes, de forma que se obtuvieron calificaciones desde " 1 " (muy inadecuado) hasta " 5 " muy adecuado. Para incluir esta variable al modelo, se generaron tres categorías (muy inadecuado / inadecuado, regularmente adecuado, y adecuado/ muy adecuado.) Para el modelo logístico la categoría referencia fue "adecuado/muy adecuado"

La información fue capturada mediante el programa Fox Pro. Para asegurar la calidad de la información se hizo doble captura, por personal estandarizado para este proceso. Para el análisis estadístico se utilizó el programa STATA 9.2

Finalmente, como complemento a este estudio, se realizó una revisión histórica documental sobre los motivos, razones y circunstancias sociales y políticas en las cuales se construyeron los puentes por sexenio presidencial desde $1960 .^{*}$

El Comité de Ética del Instituto Nacional de Salud Pública (INSP) revisó y aprobó el proyecto "Impacto de los puentes peatonales en la prevención de atropellamientos en la Ciudad de México ", a partir del cual se obtuvo la información para este artículo.

\section{Resultados}

En el Distrito Federal existen 617 puentes peatonales, clasificados en nueve categorías según el tipo de vialidad donde están colocados. El 33.5\% está sobre ejes viales, $28.4 \%$ sobre vías anulares, $12.8 \%$ sobre vías radiales; en otras categorías se encuentran los que está sobre el viaducto, vialidad principal, acceso carretero, entre otras; casi la tercera parte se encuentra en las delegaciones Iztapalapa y Gustavo A. Madero. Delegaciones como Coyoacán, Benito Juárez e Iztacalco concentran $8 \%$ en cada una de ellas.

De las 2533 entrevistas aplicadas, 68\% fueron a peatones que usaron puentes que no tenían la posibilidad de cruce a nivel de la calle, por lo que constituyeron el grupo de peatones que siempre utilizaron PP. La

\footnotetext{
* Guerra-Solalinde H. Historia y contexto de la Ciudad de México en el que se ha dado la toma de decisiones para la colocación, diseño y ubicación de los puentes peatonales entre 1952-2006. México: 2007. Documento de trabajo no publicado.
} 
muestra de peatones en los PP que tenían posibilidad de cruzar a nivel de la calle (debajo del puente) fue de 813; la prevalencia de no uso de puentes en este grupo de usuarios fue $50.5 \%$. Para fines de este estudio, se presenta el análisis de estos 813 usuarios que tuvieron posibilidad de cruce a nivel de calle.

El promedio de edad de la muestra estudiada fue 37.7 años (DE 16.3), 67\% tenían entre 15 y 44 años, y $52 \%$ eran mujeres; no se observaron diferencias para la edad entre hombres y mujeres $(p=0.089)$. Más de 50\% tenían nivel de escolaridad secundaria y preparatoria; $48 \%$ refirieron tener un trabajo de más de 20 horas a la semana; $74 \%$ de los entrevistados eran originarios del Distrito Federal, aproximadamente $94 \%$ vivía en la ciudad desde hace más de 10 años y casi $80 \%$ vivían o trabajaban cerca de la zona donde se realizó la entrevista (cuadro I). Se encontraron diferencias estadísticamente significativas en actividad laboral, frecuencia de uso y características físicas de los PP entre usuarios y no usuarios $(p<0.05)$. Se encontró una media de vivir en el DF de 32.97 años (DE 16.28 años), no se observaron diferencias estadísticas entre aquellos que se calificaron como usuarios o no usuarios $(p>0.05)$.

En relación con la movilidad en la ciudad, no se encontraron diferencias en las formas de desplazamiento en la ciudad, modo de transporte, práctica y frecuencia de manejo entre usuarios y no usuarios de $\mathrm{PP}(p>0.05)$. Es importante resaltar que, del total de la población, $93 \%$ usaba algún medio de transporte para desplazarse en la ciudad, donde los más utilizados fueron el metro, el camión, el microbús o el taxi (88.5\%); menos de la mitad (43.4\%) refirieron que sabían conducir y de éstos, $43 \%$ reportaron conducir una a tres veces por semana. En estas variables se obtuvieron tasas de no respuesta que oscilaron entre 1.23 a $7.1 \%$ (cuadro II).

Cuando se analizaron las variables relacionadas con motivos y frecuencia de uso de PP, 72.7\% de los peatones reportó usarlos generalmente, de los cuales $80 \%$ afirmaron usarlos siempre. Las principales razones para su uso fueron: "son más seguros" (80.2\%) y "no hay otra forma de cruzar" (15.8\%). Del grupo de personas que respondió que no usaba los puentes, $30.7 \%$ dijo que fue por "flojera o implica mucho esfuerzo", 25.7\% "me puedo cruzar por la calle", y 24.8\% "son inseguros". Vale la pena aclarar que los peatones que afirmaron usar los puentes, al expresar que "son más seguros", se refieren a seguridad física relacionada a no exponerse al flujo vehicular, a la afluencia de uso y al fácil acceso. Mientras, en el caso de los peatones que dijeron no usarlos porque "son inseguros", hacen referencia a los posibles riesgos relacionados con actividades delictivas (cuadro III).

El 85\% de los peatones entrevistados plantearon propuestas para promover el uso de $\mathrm{PP}$, de los cuales
$30 \%$ expresó la necesidad de mejorar la vigilancia, alumbrado y seguridad en términos de exposición a la delincuencia y $23 \%$ la de mejorar el diseño y el mantenimiento, así como difundir los beneficios de su uso (22\%) (figura 2).

En el cuadro IV se muestran los resultados del análisis con regresión logística, en el que se presentan las razones de momios cruda y ajustada para uso y no uso de PP. Los resultados ajustados muestran que los grupos de edad de 19 a 36 y de 46 y más $(R M=1.7$, IC95\% 1.06-2.86 y $R M=1.9$, IC95\% 1.14-3.33 respectivamente), muestran mayor posibilidad de no usar el PP al compararse con los menores de 19 años; no hubo diferencias estadísticamente significativas de acuerdo con la variable "escolaridad". Cuando en cuanto a características físicas el PP fue "regularmente adecuado" e "inadecuado y muy inadecuado" la probabilidad de "no uso" disminuyó 37 y $30 \%$, respectivamente, y esta diferencia resulta ser estadísticamente significativa. Ninguna de las interacciones exploradas mostró niveles de significancia estadística $(p>0.100)$.

\section{Discusión}

México, al igual que muchos países en vías de desarrollo, tiene una mezcla de usuarios que difiere del existente en países industrializados, ${ }^{2-5,9}$ donde ha quedado demostrado que el grupo de usuarios más vulnerable y a la vez el más afectado es el de los peatones. ${ }^{9-13}$

Para el año 2007 la tasa de mortalidad por atropellamientos en la Ciudad de México fue de 7.8/100.000 habitantes. Según datos de la Secretaría de Seguridad Pública (SSP) del Distrito Federal, en el año 2005 se registraron cerca de 4000 atropellamientos, de los cuales, casi $40 \%$ ocurrieron en avenidas secundarias en las cuales existen pasos peatonales. Lo anterior nos hace pensar que la infraestructura actual, dirigida a la protección (seguridad) de los peatones en la vía pública, no está siendo eficaz. En el caso de nuestro estudio, observamos que más de la mitad de los peatones no usaron el puente cuando existía posibilidad de atravesar a nivel de calle, lo que coincide con lo reportado por Mutto y colaboradores. ${ }^{14}$

Las características de la población estudiada, tanto de usuarios como de no usuarios de PP, nos indica que en su mayoría se trata de personas en edad productiva, involucrada en actividades laborales. En este estudio, más de la mitad de los peatones entrevistados reportaron no saber conducir o nunca conducir. La mayor parte de ellos tienen formas mixtas de desplazamiento en la ciudad. Está claro que se sigue desarrollando un modelo urbano que privilegia la construcción de amplias vialidades, con el fin de favorecer el desplazamiento 
Cuadro I

Características generales de los peatones usuarios y no usuarios, en PUentes Peatonales Con POSIBILIDAD de paso a nivel de Calle. MéXico, DF, 2008

Variable
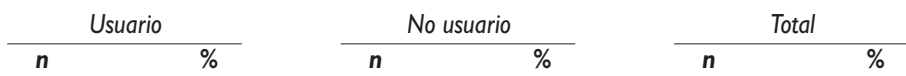

Edad (años)

\begin{tabular}{|c|c|c|c|c|c|c|}
\hline Menor de 15 & 4 & 1.0 & 3 & 0.7 & 7 & 0.9 \\
\hline 15 a 44 & 276 & 68.7 & 262 & 63.7 & 538 & 66.2 \\
\hline 45 a 64 & 98 & 24.4 & 116 & 28.2 & 214 & 26.3 \\
\hline 65 o más & 24 & 6.0 & 30 & 7.3 & 54 & 6.6 \\
\hline Total & 402 & 100.0 & $4 \mid I$ & 100.0 & 813 & 100.0 \\
\hline
\end{tabular}

Sexo

\begin{tabular}{|c|c|c|c|c|c|c|}
\hline Masculino & 192 & 47.8 & 196 & 47.7 & 388 & 47.7 \\
\hline Femenino & 210 & 52.2 & 215 & 52.3 & 425 & 52.3 \\
\hline Total & 402 & 100.0 & 411 & 100.0 & 813 & 100.0 \\
\hline
\end{tabular}

Escolaridad

\begin{tabular}{|c|c|c|c|c|c|c|}
\hline Sin estudio, primaria & 86 & 21.5 & 87 & 21.4 & 173 & 21.4 \\
\hline Secundaria & 102 & 25.4 & 94 & 23.1 & 196 & 24.3 \\
\hline Bachillerato & 136 & 33.9 & 135 & 33.2 & 271 & 33.5 \\
\hline Licenciatura/posgrado & 77 & 19.2 & 91 & 22.3 & 168 & 20.8 \\
\hline Total & 401 & 100.0 & 407 & 100.0 & 808 & 100.0 \\
\hline
\end{tabular}

Actividad laboral

\begin{tabular}{|c|c|c|c|c|c|c|}
\hline Trabaja > 20 hrs. & 184 & 45.8 & 207 & 50.5 & 391 & 48.2 \\
\hline Trabaja $<20$ hrs. & 51 & 12.7 & 31 & 7.6 & 82 & 10.1 \\
\hline No trabaja & 167 & 41.5 & 172 & 42.0 & 339 & 41.7 \\
\hline Total & 402 & 100.0 & 410 & 100.0 & 812 & 100.0 \\
\hline
\end{tabular}

Nació en el DF

\begin{tabular}{|c|c|c|c|c|c|c|}
\hline Sí & 296 & 73.6 & 307 & 75.2 & 603 & 74.4 \\
\hline No & 106 & 26.4 & $|0|$ & 24.8 & 207 & 25.6 \\
\hline Total & 402 & 100.0 & 408 & 100.0 & 810 & 100.0 \\
\hline
\end{tabular}

Vive o trabaja cerca

\begin{tabular}{|c|c|c|c|c|c|c|}
\hline Sí & 313 & 78.1 & 334 & 81.5 & 647 & 79.8 \\
\hline No & 88 & 21.9 & 76 & 18.5 & 164 & 20.2 \\
\hline Total & 401 & 100.0 & 410 & 100.0 & 811 & 100.0 \\
\hline
\end{tabular}

Frecuencia de uso de los PP, de los que sí usan $(n=582)$

\begin{tabular}{|c|c|c|c|c|c|c|}
\hline Siempre & 258 & 70.1 & 40 & 18.7 & 298 & 79.8 \\
\hline A veces & 110 & 29.9 & 174 & 81.3 & 284 & 20.2 \\
\hline \multirow[t]{2}{*}{ Total } & 368 & 100.0 & 214 & 100.0 & 582 & 100.0 \\
\hline & & & & & & $p=0.000$ \\
\hline \multicolumn{7}{|l|}{ Características físicas del PP } \\
\hline Adecuado y muy adecuado & 175 & 25.4 & 221 & 53.8 & 396 & 48.7 \\
\hline Regularmente adecuado & 125 & 31.1 & III & 27.0 & 236 & 29.0 \\
\hline Inadecuado y muy inadecuado & 102 & 43.5 & 79 & 19.2 & $|8|$ & 22.3 \\
\hline \multirow[t]{2}{*}{ Total } & 402 & 100.0 & $4 I I$ & 100.0 & 813 & 100.0 \\
\hline & & & & & & $p=0.011$ \\
\hline
\end{tabular}


Cuadro II

FoRMAS DE DESPLAZAMIENTO DE PEATONES USUARIOS Y NO USUARIOS EN PUENTES PEATONALES CON POSIBILIDAD de paso a Nivel de CALle. México, DF, 2008

Variable

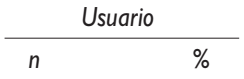

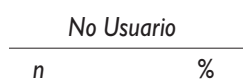

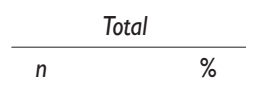

Desplazamiento en la ciudad

\begin{tabular}{|c|c|c|c|c|c|c|}
\hline Transporte & 374 & 93.0 & 381 & 92.7 & 755 & 92.9 \\
\hline Caminando & 28 & 7.0 & 30 & 7.3 & 58 & 7.1 \\
\hline \multirow[t]{2}{*}{ Total } & 402 & 100.0 & 411 & 100.0 & 813 & 100.0 \\
\hline & & & & & & 0.853 \\
\hline
\end{tabular}

Modo de transporte

\begin{tabular}{|c|c|c|c|c|c|c|}
\hline Taxi/micro y masivos & 323 & 86.4 & 345 & 90.6 & 668 & 88.5 \\
\hline Auto particular & 51 & 13.6 & 36 & 9.4 & 87 & 11.5 \\
\hline Total & 374 & 100.0 & 381 & 100.0 & 755 & 100.0 \\
\hline
\end{tabular}

Sabe manejar

\begin{tabular}{|c|c|c|c|c|c|c|}
\hline Sí & 173 & 43.4 & 185 & 45.8 & 358 & 44.6 \\
\hline No & 226 & 56.6 & 219 & 54.2 & 445 & 55.4 \\
\hline Total & 399 & 100.0 & 404 & 100.0 & 803 & 100.0 \\
\hline
\end{tabular}

Frecuencia de manejo, sólo para los que saben manejar $(n=353)$

\begin{tabular}{|c|c|c|c|c|c|c|}
\hline Diario & 64 & 37.4 & 57 & 31.3 & $12 \mid$ & 34.3 \\
\hline I-3 veces por semana & 69 & 40.4 & 84 & 46.2 & 153 & 43.3 \\
\hline Casi nunca/nunca & 38 & 22.2 & 41 & 22.5 & 79 & 22.4 \\
\hline Total & $17 \mid$ & 100.0 & 182 & 100.0 & 353 & 100.0 \\
\hline
\end{tabular}

Cuadro III

Razones de uso y no uso de puentes peatonales con posibilidad de paso a nivel de calle. México, DF, 2008

Variable

Razones de no uso

\begin{tabular}{l} 
Son inseguros \\
\hline Me puedo cruzar por la calle \\
\hline No puedo hacerlo \\
\hline Otra razón \\
\hline Total
\end{tabular}

Razones de uso

\begin{tabular}{|c|c|c|c|c|c|c|}
\hline Son más seguros & 224 & 80.3 & 233 & 80.1 & 457 & 80.2 \\
\hline No hay otra forma de cruzar & 42 & 15.0 & 48 & 16.5 & 90 & 15.8 \\
\hline Porque puedo hacerlo & 13 & 4.7 & 10 & 3.4 & 23 & 4.0 \\
\hline Total & 279 & 100.0 & 291 & 100.0 & 570 & 100.0 \\
\hline
\end{tabular}

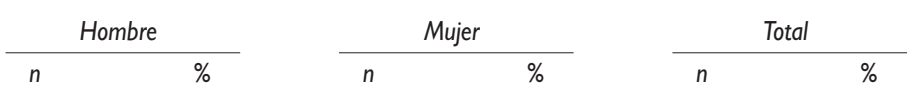

\begin{tabular}{rrrrrr}
23 & 22.5 & 31 & 26.7 & 54 & 24.8 \\
31 & 30.4 & 25 & 21.5 & 56 & 25.7 \\
32 & 31.4 & 35 & 30.2 & 67 & 30.7 \\
\hline 8 & 7.8 & 22 & 19.0 & 30 & 13.8 \\
\hline 8 & 7.8 & 3 & 2.6 & 11 & 5.0 \\
102 & 100.0 & 116 & 100.0 & 218 & 100.0 \\
\hline
\end{tabular}




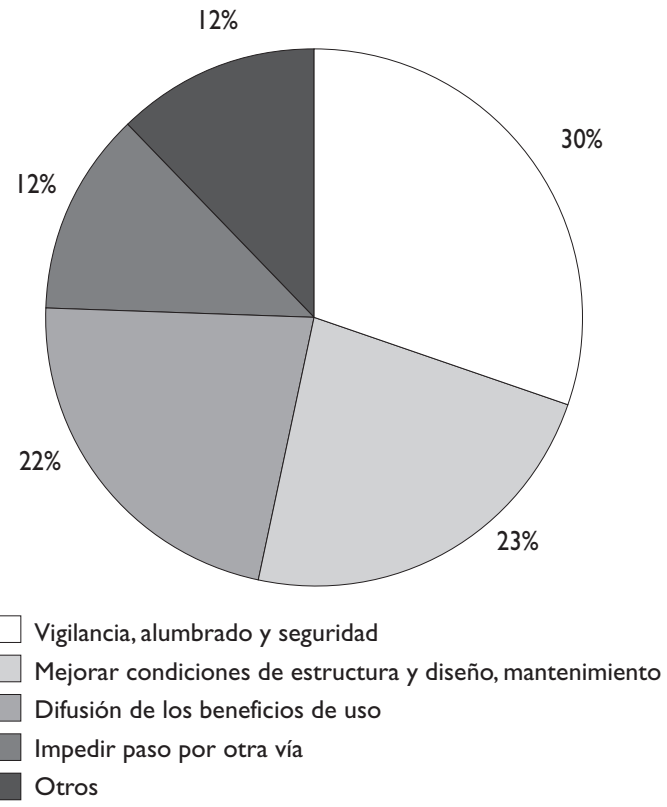

Figura 2. Propuestas de los peatones para promover el uso de los puentes peatonales en el Distrito Federal, 2008

y la movilidad de vehículos de motor, $^{2}$ lo que obliga al peatón a utilizar el espacio diseñado para éstos, ya sea por razones de inseguridad de los PP, o porque no existen otras formas de cruzar la calle, y asume el riesgo que significa enfrentar el flujo vehicular.

Contrario a lo reportado en otros escenarios, ${ }^{15}$ la documentación realizada por Guerra-Solalinde identificó que los PP no han sido construidos principalmente para prevenir atropellamientos sino para mejorar el movimiento de los vehículos que se desplazan bajo los mismos, por ende, su construcción tiene una lógica más de ingeniería urbana que de prevención de lesiones. Lo anterior deja claro el hecho de que los PP podrían constituirse como una medida eficaz para hacer más segura la movilidad de los grandes volúmenes de peatones que se desplazan en grandes ciudades como el DF. Sin embargo, constituyen un sitio de exposición para la ocurrencia de atropellamientos.

Los responsables de la planeación urbana tendrán que reflexionar sobre la utilidad de los PP, los cuales, además de no cumplir con la función de separar de forma segura los espacios entre los usuarios de la vía pública, han traído la exposición a otros riesgos sobre la salud como la "violencia urbana" (asaltos, riñas, violencia sexual, etc.), sin olvidar los peligros derivados de la falta de mantenimiento de su estructura. Todo lo
Cuadro IV

Regresión logística multivariada para uso Y No uso de PUentes Peatonales. MéXICO, DF, 2008

\begin{tabular}{|c|c|c|}
\hline \multirow[b]{2}{*}{ Variables } & \multicolumn{2}{|c|}{ No uso de PP } \\
\hline & RM cruda (IC 95\%) & RM ajustada \\
\hline
\end{tabular}

Grupos de edad

\begin{tabular}{lcc} 
Menor de 19 años & I & I \\
\hline 19 a 36 años & $1.8(1.10-2.91)^{*}$ & $1.7(1.06-2.86)^{*}$ \\
\hline 37 a 45 años & $1.2(0.68-2.05)^{N / 5}$ & I.I $(0.63-1.96)^{N / 5}$ \\
\hline 46 y más & $1.9(1.17-3.22)^{*}$ & $1.9(1.14-3.33)^{*}$ \\
\hline
\end{tabular}

\begin{tabular}{|c|c|}
\hline \\
\hline \multicolumn{2}{|l|}{$\begin{array}{l}\text { Escolaridad } \\
\text { Sin instrucción y primaria }\end{array}$} \\
\hline Sin instrucción y primaria & $0.91(0.60-1.37)^{\mathrm{N} / \mathrm{S}} \mathrm{I} .06(0.69-1.63)^{\mathrm{N} / \mathrm{S}}$ \\
\hline Bachillerato & $0.98(0.67-I .43)^{\mathrm{N} / \mathrm{S}} \quad \mathrm{I} . \mathrm{I}(0.74-\mathrm{I} .68)^{\mathrm{N} / \mathrm{S}}$ \\
\hline Licenciatura y posgrado & I.I $(0.76-1.78)^{\mathrm{N} / \mathrm{S}} \quad$ I..2 (0.78-I.92) N/S \\
\hline
\end{tabular}

Características físicas del PP

\begin{tabular}{lcc}
\hline Adecuado y muy adecuado & I & I \\
\hline Regularmente adecuado & $0.61(0.43-0.87)^{*}$ & $0.63(0.44-0.91)^{*}$ \\
\hline Inadecuado y muy inadecuado & $0.70(0.50-0.97)^{*}$ & $0.70(0.50-0.98)^{*}$
\end{tabular}

Log likelihood $=-549.75838$

$* p<0.05$

N/S: No significativo

anterior tiene correlación directa con las sugerencias que hacen los peatones respecto de las medidas a considerar para promover su uso.

La necesidad de mejorar la seguridad y garantizar la integridad física de los peatones hacen necesaria la implementación de medidas de prevención que favorezcan la recuperación del espacio público que garantice el acceso libre y seguro de los mismos a toda la infraestructura urbana. ${ }^{13,14,16}$ Lo anterior se complementa con el componente de la educación, tanto de conductores como de peatones, sobre la necesidad de promover comportamientos y actitudes de respeto mutuo. $^{16}$

La metodología para determinar el tamaño de muestra en aquellos casos donde existe un gran volumen de usuarios en espacios no cautivos y donde se quiere medir objetivamente una característica que tiene que ver con comportamiento (uso y no uso), teniendo en consideración características tan importantes como grupos poblacionales y sexo, representó uno de los grandes 
retos del presente trabajo, ya que no se encontraron estudios en el campo de las lesiones en vía pública que den cuenta de este componente.

Consideramos necesario insistir en la definición y puesta en marcha de una política pública que fortalezca las capacidades de los gobiernos para garantizar e impulsar la movilidad de una forma segura, equitativa, saludable y sustentable para todos y cada uno de los actores que comparten día a día la vía pública.

\section{Agradecimientos}

Este trabajo se realizó gracias al apoyo del Consejo Nacional de Ciencia y Tecnología al proyecto 13880.

\section{Declaración de conflicto de intereses}

Declaramos no tener conflicto de intereses.

\section{Referencias}

I. Mohan D. Injury control and safety promotion: ethics, science and practice. En: Mohan, D, Tiwari G (eds). Injury prevention and control. Londres: Taylor \& Francis, 2000:I-12.

2. Hijar M. El crecimiento urbano y sus consecuencias no planeadas. El caso de los atropellamientos. En: Knaul F, Nigenda G. (eds.) Caleidoscopio de la Salud, México: FUNSALUD,2003:89-97.
3. Hijar M, Troste J, Bronfman M. Pedestrian injuries in México: a multimethod approach. Soc Sci Med 2003; 57(II):2149-2159.

4. Hijar M, Kraus J, TovarV, Carrillo C.Analysis of fatal pedestrian injuries in Mexico City 1994-1997. Injury Int J Care Injured 200 I;(32):279-284. 5. Hijar M,Vasquez-Vela E,Arreola-Rissa C. Pedestrian traffic injuries in México. Inj Control Saf Promot 2003;10(3):37-43.

6. Dawson-Saunders B, Trapp RG. Bioestadística Médica $.2^{\mathrm{a}}$ ed. México: Manual Moderno, 1996

7.Argimón-Pallas J, Jiménez-Villa J. Métodos de investigación aplicados a la atención primaria de salud. $2^{\mathrm{a}}$ ed. Barcelona: Mosby-Doyma, 1994

8. Kleinbaum DG, Kupper LL, Morgenstern H. Epidemiologic research. Principles and quantitative methods. Belmont California: Lifetime Learning Publications. Wadsworth, 1982.

9. Hazen A, Ehiri J. Road Traffic Injuries: hidden epidemic in less developed countries. J Natl Med Assoc 2006;98(I):73-82.

10. Mohan D. Traffic safety and city structure: lessons for the future. Salud Publica Mex 2008; 50(SI):93-100.

II. Forjouh S. Traffic related injury prevention interventions for low countries. Inj Control Saf Promot 2003; 10 (3): 109-II 8.

12.Tiwari G, Mohan D, Fazio J. Conflict Analysis for prediction of fatal crash locations in mixed traffic stream. Accident Anal Prev 1998;30:207-215.

13. Echeverry J, Mera J], Villota J, Zárate C. Actitudes y comportamientos de los peatones en los sitios de alta accidentalidad en Cali, Colombia. Med 2005;36(2):79-84.

14. Mutto M, Kobusingye O, Lett R. The effect of an overpass on pedestrian injuries on a major highway in Kampala. Afr Health Sci 2002; 2(3) 89-93.

15. Secretaría de Gobierno - Alcaldía Mayor de Bogotá. La accidentalidad vial en Bogotá DC - Colombia. Mayo de 2002

16. Asociación de peatones de Quito. La ciudad y los peatones. Ia . edición. 2008. [Consultado 23 julio 2009]. Disponible en: www.peatones.org. 